\title{
Re: Predicting High-Grade Cancer at Ten-Core Prostate Biopsy Using Four Kallikrein Markers Measured in Blood in the ProtecT Study
}

\author{
Richard J. Bryant, Daniel D. Sjoberg, Andrew J. Vickers, Mary C. Robinson, Rajeev Kumar, \\ Luke Marsden, Michael Davis, Peter T. Scardino, Jenny Donovan, David E. Neal, Hans Lilja, \\ Freddie C. Hamdy
}

University of Oxford, Department of EpidemiologyetBiostatistics and Nuffield Department of Surgical Sciences Memorial Sloan Kettering Cancer Center, New York, USA

JNCl J Natl Cancer Inst (2015) 107(7): djv095 doi:10.1093/jnci/djv095

\section{EDITORIAL COMMENT}

An elevated prostate specific antigen (PSA) level is not usually related with aggressive (Gleason 7 or higher) prostate cancer (PCa). Some PCa biomarker tests have been developed for refining patient selection for biopsies. A statistical model (4Kscore) based on measurement of free, intact and total PSA, and kallikreinrelated peptidase 2 combined with patient age, digital rectal examination and prior biopsy status was developed for this purpose. The 4Kscore tests recently recommended for both patients with prior biopsy and prior negative biopsy in 2015 NCCN Guidelines for Prostate Cancer Early Detection (1).

In the present study, the four kallikreins (4K) enhanced prostate cancer detection compared with PSA and age alone. Area under the curve (AUC) for the 4K was 0.719 (95\%, Cl: $0.704-0.734)$ vs. 0.634 (95\%, Cl: 0.617-0.651, $\mathrm{p}<0.001)$ for PSA and age alone for any-grade cancer, and 0.820 (95\%, Cl: 0.802-0.838) vs. 0.738 (95\%, Cl: 0.716-0.761) for high-grade cancer. Using a 6\% risk of high-grade cancer as an illustrative cutoff, for 1000 biopsied men with PSA levels of $3.0 \mathrm{ng} / \mathrm{mL}$ or higher, the model would reduce the need for biopsy in 428 men, detect 119 high-grade cancers, and delay diagnosis of 14 of 133 high-grade cancers. Developed models exhibited excellent discrimination on independent validation among men with only serum samples available for analysis. It is important to remember for both physician and patient that there is no cut-off threshold either to perform or not to perform yet.

4Kscore test is a promising statistical model for detecting aggressive PCa. Beside age, DRE, and biopsy status additional clinical features (family history/genetics, body mass index and race etc.) to the model may improve detecting clinically significant PCa.

\section{REFERENCE}

1. http://www.nccn.org/professionals/physician_gls/f_guidelines.asp\#prostate_detection 